\title{
Escribir para aprender en dos disciplinas: construcción conjunta del conocimiento y extensión del tiempo didáctico
}

\author{
María Elena Molina ${ }^{1}$ \\ Orcid: 0000-0002-1663-4652 \\ Laura Marina Colombo² \\ orcid: 0000-0001-6026-4436
}

\section{Resumen}

En las últimas décadas, los vínculos entre la escritura y el aprendizaje han sido ampliamente estudiados. En la enseñanza superior, en particular, los investigadores se han centrado en las producciones de los estudiantes, en sus puntos de vista respecto de la escritura en las diferentes disciplinas y en las complejas relaciones entre los contenidos de las disciplinas y las prácticas de escritura, entre otros. Sin embargo, escasas investigaciones se centran en las prácticas docentes que entrelazan la escritura y los contenidos disciplinarios. Este trabajo analiza dos aspectos claves de las prácticas de enseñanza cuando se escribe para aprender: el tiempo didáctico y las responsabilidades en la construcción del conocimiento. Los resultados forman parte de una investigación didáctica diseñada como un estudio de casos múltiples en dos disciplinas universitarias (Lingüística y Biología). Los casos estudiados pertenecen a dos cursos de primer año impartidos en dos de las principales universidades argentinas. Las técnicas de recolección de datos incluyeron, principalmente, análisis de documentos, observaciones de clase y entrevistas semiestructuradas con los estudiantes. En este sentido, centrándose en las observaciones de clase, este trabajo muestra cómo se amplió el tiempo de enseñanza en esos cursos al entrelazar las prácticas de enseñanza con la escritura como herramienta de aprendizaje. En estas clases, la escritura prolongó el tiempo didáctico en la medida en que los estudiantes tuvieron la oportunidad de escribir fuera de las aulas y de discutir lo que escribieron dentro de ellas. Además, esta práctica ayudó a estudiantes y profesores a compartir, de manera más simétrica, las responsabilidades vinculadas con la construcción del conocimiento.

\section{Palabras clave}

Prácticas de enseñanza - Contenidos disciplinares - Lingüística - Biología - Didáctica del nivel superior.

1- CONICET. Universidad Nacional del Sur, Bahía Blanca, Argentina. Contact: elena.molina@uns.edu.ar.

2- CONICET. Universidad de Buenos Aires, Buenos Aires, Argentina. Contact: laura.colombo@conicet.gov.ar. 


\section{Writing to learn in two disciplines: negotiating knowledge-construction and extending teaching-time}

\section{Abstract}

In the last decades, the links between writing and learning have been widely studied. In higher education, researchers have focused on students' productions, on their standpoints towards writing in different disciplines, and on the complex relations between subject contents and writing practices, among others. However, teaching practices that intertwine writing and disciplinary contents have received scarce attention. This work emphasises two key aspects of teaching practices related to writing to learn activities: teaching time and responsibilities in knowledge-construction. A multiple case study was conducted with two first-year courses, one in Linguistics and the other in Biology. These classes were offered in two public universities in Argentina. Data collection techniques included, mainly, classroom documents, class observations and semi-structured interviews with students. Focusing on class observations, this paper shows how the teaching time was extended by intertwining teaching practices with writing as a learning tool. In both courses, writing extended class-time when students had the opportunity to write outside the classrooms and to discuss what they wrote in whole-class discussions. Additionally, these uses of writing helped students and professors to share, in a more symmetrical way, the responsibilities towards knowledge construction.

\section{Keywords}

Teaching practices - Disciplinary contents - Linguistics - Biology - Teaching in higher education.

\section{Introducción ${ }^{3}$}

¿Qué pasa con el tiempo de enseñanza y la construcción del conocimiento cuando uno escribe para aprender en asignaturas universitarias? Este trabajo busca caracterizar las formas en que la escritura, como objeto de aprendizaje y como herramienta de enseñanza (MOLINA Y CARLINO, 2019), afecta la gestión del tiempo didáctico y la distribución de las responsabilidades en la construcción de conocimientos en dos cursos universitarios argentinos de dos disciplinas: Lingüística y Biología.

La función epistémica de la escritura ha sido ampliamente estudiada en las últimas décadas. Muchos investigadores han profundizado en la forma en que la escritura puede utilizarse como instrumento de elaboración de conocimientos cuando se enseñan diferentes

3- Agradecimientos: Este trabajo se realizó en el marco de un proyecto de investigación (PICT-2014-2793) financiado por la Agencia Nacional de Promoción Científica y Tecnológica (ANPCYT) de Argentina. 
disciplinas y en distintos niveles educativos. Por un lado, los autores anglosajones han sido pioneros en investigar la lectura y la escritura en la educación superior. Estas indagaciones se diferenciaron de paradigmas de enseñanza que concebían la escritura como una habilidad de comunicación básica y general. Así, partieron de la idea de que las dificultades de los estudiantes en lo que concierne a la lectura y la escritura no eran simplemente el resultado de déficits o fracasos individuales en los niveles previos de enseñanza (BAZERMAN ET AL., 2005). Lejos de ello, los estudios realizados en el marco de "Escribir a través del currículum", "Escribir y leer para aprender" y "Escribir en las disciplinas" han mostrado que la lectura y la escritura pueden ser herramientas poderosas que median la elaboración de conocimientos sociales (BAZERMAN, 1988), el aprendizaje de contenidos disciplinarios (LANGER Y APPLEBEE, 1987) y las formas de hacer y pensar en las disciplinas (CARTER, FERZLI Y WIEBE, 2007). Actualmente, estas líneas de investigación continúan analizando los desafíos de la lectura y la escritura en el ámbito universitario (CONDON Y RUTZ, 2012; RUSSELL, 2013; THAISS Y PORTER, 2010).

Por otro lado, en Argentina, diferentes líneas de investigación se han venido ocupando de los desafíos que enfrentan los estudiantes universitarios en materia de lectura y escritura. Desde una perspectiva lingüística, prevalecieron los estudios diagnósticos. Estos trabajos detallaron las dificultades de los estudiantes para comprender y escribir diferentes géneros académicos como artículos científicos, reseñas, ensayos, monografías y disertaciones (ARNOUX ET AL., 1996; ARNOUX Y ALVARADO, 1997; PIACENTE Y TITTARELLI, 2003). Adicionalmente, otros investigadores se enfocaron en el diseño e implementación de cursos y/o talleres de lectura y escritura académicas implementados por distintas universidades con el fin de superar dichos problemas (FERNÁNDEZ, IZUZQUIZA Y LAXALT, 2004; NATALE, 2004). En algunos de estos trabajos, se analizaron las producciones escritas de los estudiantes para describir con mayor detalle sus avances y dificultades (DI STEFANO Y PEREIRA, 2004).

Relacionado con esto, pero desde un enfoque diferente y menos difundido, existe un conjunto de trabajos argentinos que estudian las situaciones docentes que se dan en las aulas. Estas iniciativas de investigación-acción han analizado las condiciones de enseñanza que promueven el aprendizaje de los contenidos disciplinares a través de la lectura y la escritura (VÁZQUEZ, JAKOB, PELLIZA Y ROSALES, 2003; PADILLA, AVILA Y LOPEZ, 2007; IGLESIA Y DE MICHELI, 2009; PADILLA, 2012). Otra línea de investigación argentina se ha centrado en las perspectivas de los estudiantes y los profesores sobre la escritura. Sus resultados han puesto de manifiesto los desajustes que suelen existir entre la comprensión que tienen los estudiantes y los profesores acerca de la distribución de responsabilidades, incluyendo quiénes deben saber ya escribir -estudiantes-, y quiénes tienen que enseñar a hacerlo -profesores- (ALVARADO Y CORTÉS, 2000; CARLINO, 2002, 2007; VÁZQUEZ Y MIRAS, 2004). En este sentido, el equipo de investigación GICEOLEM ${ }^{4}$ (Grupo para la Inclusión y la Calidad Educativas a través de Ocuparnos de la Lectura y la Escritura en todas las Materias) plantea que los estudios diagnósticos por sí solos no

4- GICEOLEM (Grupo para la Inclusión y la Calidad Educativas a través de Ocuparnos de la Lectura y la Escritura en todas las Materias), equipo de investigación dirigido por la Dra. Paula Carlino (CONICET), con base en el Instituto de Lingüística de la Universidad de Buenos Aires [https://sites. google.com/site/giceolem2010/who-are-we]. 
proporcionan una visión adecuada de la complejidad de las prácticas sociales de la lectura y la escritura en la educación superior. Por esta razón, varios trabajos se han centrado no sólo en los textos producidos por los estudiantes, sino también en lo que los estudiantes reportan sobre sus producciones y los desafíos que enfrentan (CARTOLARI Y MOLINA, 2016; COLOMBO, 2018; ROSLI, CARLINO Y CARTOLARI, 2013). También se considera esencial incluir como objeto de estudio lo que sucede en diversos entornos institucionales, con diferentes profesores y enfoques de enseñanza en los niveles secundario y superior. De este modo, pues, en este artículo se explora lo que sucede con el tiempo didáctico y la construcción de conocimientos en el aula cuando los estudiantes escriben para aprender en dos disciplinas: Lingüística y Biología.

Nuestro marco teórico se basa en la Didáctica como disciplina que estudia sistemáticamente las prácticas de enseñanza (CAMILLONI, 2012; SENSEVY, 2011). Desde una perspectiva lingüística, concebimos la escritura como una práctica social (BAZERMAN Y PRIOR, 2004; BARTON Y HAMILTON, 1998; LEA Y STREET, 1998; CARLINO, 2005, 2013; ENTRE OTROS) y, en lo que respecta a la escritura académica, en particular, la entendemos como una construcción argumentativa (PADILLA, 2012). Por lo tanto, asumimos que en la educación superior los estudiantes necesitan aprender a ejercer las prácticas discursivas y textuales inherentes a sus disciplinas.

Desde una perspectiva didáctica, las categorias que guiaron nuestro análisis fueron los conceptos de contrato y medio (BROUSSEAU, 2007) y el de cronogénesis (es decir, la génesis del tiempo didáctico o tiempo de enseñanza) (SENSEVY, 2009, 2011). Según Brousseau (2007), el contrato didáctico es un sistema de expectativas entre profesores y estudiantes que se basa en los conocimientos disciplinares en juego. El medio didáctico tiene una doble caracterización: a) el sistema antagonista con el que el estudiante interactúa; y b) el contexto cognitivo de la acción, es decir, los conocimientos y las experiencias previas de los estudiantes. Utilizamos la noción de cronogénesis o "tiempo didáctico" para describir la progresión de los contenidos en el tiempo, progresión que es propuesta por el profesor y abordada por los estudiantes en la acción conjunta (SENSEVY, 2007).

\section{Metodología}

La presente investigación se diseñó como un estudio de casos múltiples (CRESWELL, 2007; MAXWELL, 2005; STAKE, 1998) y desde un enfoque didáctico naturalista (ARTIGUE, 1990; RICKENMANN, 2006). Nuestro estudio seleccionó y estudió en profundidad dos casos que presentaban iniciativas didácticas innovadoras. Estos casos consistían en dos cursos universitarios introductorios ofrecidos en dos instituciones públicas argentinas. Estos cursos fueron seleccionados porque sus profesores integraron deliberadamente la escritura en sus prácticas de enseñanza cotidianas.

El caso Lingüística corresponde a un curso introductorio de primer año sobre Estudios del Discurso, con un fuerte componente de producción y comprensión textual. Se ofrece a los estudiantes de Licenciatura y Profesorado en Letras de una de las principales universidades públicas argentinas. Este curso -de 4 horas semanales- se reúne dos veces por semana durante todo un año y es impartido por profesores con formación académica en 
Lingüística, Escritura y Retórica, que también están interesados en Pedagogía y Didáctica. Durante el primer cuatrimestre, los estudiantes aprenden sobre géneros discursivos, discurso científico, escritura académica y argumentación. Se espera que asistan a clases plenarias y prácticas. Alrededor de 200 estudiantes se matriculan cada año y asisten a las clases plenarias. Para las clases prácticas, se conforman grupos más pequeños, de 30 a 50 estudiantes. Durante el primer cuatrimestre, trabajan varios géneros discursivos, incluyendo los géneros cotidianos (editoriales, noticias, publicidad, cuentos, etc.) y los académicos (ponencias, artículos, capítulos de libros, reseñas, etc.). Durante el segundo cuatrimestre, se les pide que escriban una ponencia relacionada con uno de los géneros enseñados durante la primera parte del año. Pueden trabajar solos o en grupos de 2 a 4 estudiantes, siendo esto último lo más recomendado por los docentes. Para apoyar este proceso, durante el segundo cuatrimestre, las clases prácticas se convierten en reuniones semanales de tutoría. Hacia el final del curso, los estudiantes presentan su trabajo en un evento científico organizado por la universidad.

Por otro lado, el caso Biología es una clase introductoria para los estudiantes inscriptos en las carreras de Medicina, Veterinaria, Psicología y Ciencias del Medio Ambiente, entre otras. Este curso cuatrimestral es ofrecido por una de las principales universidades públicas argentinas. La clase se reúne durante tres horas, dos veces por semana. Alrededor de 80 a 100 estudiantes cursan la materia. El curso es co-enseñado por dos profesoras con formación académica en Biología, pero que también están interesadas en el enfoque de leer y escribir para aprender. En este curso, los estudiantes se enfrentan regularmente a tareas de escritura basadas en problemas sobre temas biológicos. Por lo tanto, a menudo se les pide que lean con el propósito de responder a preguntas inferenciales y argumentar a favor o en contra de diferentes ideas e interpretaciones. Todas las preguntas tienen como objetivo relacionar los conceptos biológicos con situaciones prácticas de la vida real. Los estudiantes suelen leer y escribir en casa y comparten sus producciones en discusiones plenarias que ocupan la mitad del tiempo de clase.

Los datos fueron recogidos durante un cuatrimestre. En cuanto al caso Lingüística, además de las clases, también se observó y se grabó el evento científico organizado por la universidad (16 horas en total). Durante este evento, los estudiantes presentaron y defendieron sus ponencias frente a compañeros y profesores. En este artículo, trabajamos sobre los registros de esta instancia de exposición. En el caso Biología, se observaron y grabaron las 27 clases ofrecidas durante todo el cursado (81 horas en total). Después de un análisis preliminar, se seleccionaron 6 clases dedicadas a la enseñanza de una unidad sobre Expresión Genética (18 horas en total). Se eligió esta unidad porque, en comparación con otras, los estudiantes entregaron con mayor frecuencia textos argumentativos cortos y los reescribieron a partir de la retroalimentación de las profesoras. El análisis de los datos se basó en la propuesta de diseño cualitativo-interactivo de Maxwell (2005). Analizamos las transcripciones utilizando dos estrategias fundamentales: la contextualización y la codificación (MAXWELL Y MILLER, 2008), buscando relaciones de contigüidad y similitud entre los datos. 


\section{Resultados}

Nuestros resultados muestran que, en ambos cursos universitarios, el entrelazamiento entre las prácticas de escritura y las prácticas de enseñanza influyó no sólo en quiénes asumieron la responsabilidad en la construcción de conocimientos, sino también en cómo se gestionó el tiempo didáctico. Por un lado, profesores y estudiantes compartieron la responsabilidad en la construcción del conocimiento: este no fue una prerrogativa de los profesores, sino una construcción conjunta con sus estudiantes. Por otra parte, el tiempo didáctico se amplió gracias a la inclusión de la escritura en las clases, ya que los estudiantes escribieron en casa, pero para luego compartir y discutir sus textos con compañeros y docentes. De este modo, la acción conjunta superó los límites temporales y espaciales del aula. Esta acción se inició de forma individual, con cada estudiante produciendo sus textos en sus hogares y continuó después en el espacio y el tiempo del aula, con los profesores organizando las actividades en torno a las producciones escritas de los estudiantes.

La ampliación del tiempo didáctico y la renegociación de las responsabilidades acerca de la producción de conocimientos tuvo lugar en estos dos casos porque se cumplieron algunas condiciones de enseñanza. En primer lugar, en ambos casos, las tareas de escritura solicitaron una escritura argumentativa. En el caso Lingüística, los estudiantes precisaron escribir como lo hacen los expertos al producir una ponencia. Adicionalmente, la presentaron en un evento científico auténtico. En el caso Biología, las tareas de escritura se utilizaron para acercarse y comprender mejor los conceptos disciplinares. Las tareas siempre incitaban a los estudiantes a razonar y justificar sus respuestas. Además, el trabajo en clase se organizó en torno a actividades de revisión (i.e., comentar y debatir lo leído y lo escrito) que usualmente ocupaban casi la mitad del tiempo total de la clase. Cabe destacar que lo que caracterizamos como la ampliación del tiempo didáctico en estos casos no se basa sólo en la "asignación de tareas" (CARLINO ET AL., 2013), ni en pedir a los alumnos que "se las arreglen solos". Al contrario, nos referimos a que los profesores dedicaron tiempo de clase a la escritura y la llevaron al interior del aula. De este modo, toda la escritura hecha fuera del aula tuvo un significado genuino para los estudiantes. Se convirtió en un hilo conductor dentro del aula y en un eje vertebrador entre las diferentes clases. La segunda condición de enseñanza consistió en la reticencia de los profesores y el carácter dialógico de sus intervenciones. Según Sensevy (2011), la reticencia radica en la omisión voluntaria por parte del profesor de lo que podría o debería decirse para que los alumnos puedan actuar de forma autónoma. En las clases de Lingüística y Biología, los profesores propusieron tareas de escritura argumentativa que planteaban retos a los estudiantes. Intentaron mantener este desafío a través de intervenciones dialógicas que devolvieran el problema a los estudiantes y lo mantuvieran en lugar de resolverlo de inmediato.

En las siguientes secciones, presentamos algunos ejemplos de situaciones que sucedieron comúnmente en estas dos clases. Las dos situaciones distintivas que se presentan a continuación son: "Defender textos frente a pares y docentes" (Lingüística) y "Discutir textos frente a pares y docentes" (Biología). 


\section{Situación de defensa de textos frente a pares y docentes (caso Letras)}

Dado que la "situación de clase" puede adoptar muchas formas diferentes (DAVINI, 2008), en el caso Lingüística, en lugar de centrarnos en lo que ocurrió en el aula, buscamos comprender cómo dialogaron los profesores, los estudiantes y los conocimientos en un momento clave de este curso: la presentación y defensa de las ponencias. La situación de "Defensa de textos frente a pares y docentes" se produjo después de que los estudiantes presentaran sus ponencias en las jornadas organizadas por la universidad. Es decir, después de sus presentaciones orales, los estudiantes-expositores respondieron preguntas del público. Esta situación fue muy importante ya que constituyó el núcleo de todo el trabajo realizado a lo largo del cursado. El proceso de convertirse en autores de sus propios trabajos, que llevó un año de trabajo con sus tutores, culminó en esta situación. De esta manera, tuvieron una primera experiencia de escritura académica en la comunidad disciplinar de los Estudios del Discurso y esto implicó no sólo la textualización de ciertas ideas, sino también el someterlas a las preguntas, los comentarios y las opiniones de sus compañeros. En lo que sigue, analizamos un fragmento de un diálogo posterior a una presentación.

En este intercambio, Ramiro, un estudiante que escribió su trabajo de forma individual, defiende su trabajo titulado La alegría y la tristeza en la geografía literaria de Alejandro Nicolau. Allí, analiza los cuentos de Alejandro Nicolau, escritor contemporáneo de Tucumán (Argentina), a quien pudo entrevistar.

[4] Estudiante 2: Así que haces una relación, según lo que veo, con esta autora coreana. ¿Cómo lo haces? ¿Por qué?

[5] Ramiro: Sí, ella es una autora coreana. Hay espacio y emociones... En nuestro trabajo fue difícil no caer en interpretaciones psicológicas. Así que quise establecer algunas comparaciones entre lo que hice y lo que esta autora afirma. No queríamos, mi alter ego y yo [risa generalizada porque Ramiro trabajó solo], profundizar en las motivaciones o las estructuras psíquicas a partir de la descripción del espacio. Al contrario, [queríamos] tomar la alegría y la tristeza, dos emociones que están entre las cinco consideradas básicas en la Psicología, pero queríamos hacer ese análisis desde un punto de vista literario. Es decir, desde el punto de vista del texto y del autor, no desde el punto de vista psicológico. Por lo tanto, volviendo a tu pregunta, la comparamos con una obra que viene del análisis literario, alejándose de la Psicología, por eso [elegimos] la autora coreana que trabaja desde un enfoque literario.

[6] Profesora Silvana: Lo único que yo mencionaría es que hay que atenuar un poco este establecimiento del nicho porque creo que, desde el campo de la Semiótica y los análisis semióticos de la literatura, todos los enfoques tienen en cuenta la categoría "espacio" y hacen un análisis que va más allá del psicológico. Por ejemplo, pienso en los cuentos de Ana María Matute, "Pecado de omisión", donde los personajes se analizan en relación con los espacios que habitan. Y estos son análisis frecuentes desde una perspectiva semiótica. Por lo tanto, tu nicho, la forma en que lo escribiste, es muy categórica. Creo que deberías atenuarlo porque no es que el espacio no haya sido estudiado como un valor simbólico. Desde la teoría literaria, el espacio ha 
sido estudiado como un valor simbólico. Vos, por supuesto, le da una visión interesante y muy significativa. Incluso quería que me enseñara lo que escribe Méndez, que citás allí.

[7] Profesora Emilia: Hay una diapositiva al respecto [Ramiro busca en su Power Point y asiente con la cabeza].

[8] Profesora Silvana: [Observando la búsqueda del estudiante en el Power Point] Antes, antes, debe ser cuando mostrás las referencias. Quiero ver lo que escribe Méndez, porque me llamó la atención cuando lo citaste.

[9] Ramiro: "Hacia una teoría del signo espacial en la ficción narrativa contemporánea”. Es un artículo de investigación.

[10] Profesora Silvana: "Hacia una teoría del signo espacial en la ficción narrativa contemporánea".

[11] Ramiro: Sí, sí [mirando su computadora y echando un vistazo a sus notas].

[12] Profesora Silvana: Sí, era sólo una cuestión de matiz, nada más, que creo que se puede atenuar un poco porque, tal vez desde otras perspectivas, se ha explorado el espacio con valor simbólico en las obras literarias.

[13] Ramiro: Sí, quería sobre todo subrayar, digamos, que el aspecto simbólico no sería central en mi obra, sino la emoción que proviene del aspecto simbólico. Como dije, es una especie de resumen de varias cosas. Pero es cierto que hay obras que no hemos tenido en cuenta.

Como puede verse en el extracto anterior, hay una progresión del conocimiento basada en la presentación de Ramiro. El Estudiante 2, la profesora Silvana y la profesora Emilia intervinieron pidiendo más información, ofreciendo sugerencias y solicitando una justificación. En esta situación, los profesores dieron a los estudiantes la posibilidad de convertirse en autores e incipientes investigadores sobre un género discursivo de su elección, incitándolos a responsabilizarse de sus afirmaciones y del conocimiento particular construido en torno a su investigación. Este proceso fue andamiado en las sesiones de tutoría durante un período de cuatro meses. Al final del cuatrimestre, cada estudiante o grupo de estudiantes entregó entre 10 y 15 borradores de su trabajo y todos ellos fueron comentados por los profesores-tutores. No obstante, fue responsabilidad exclusiva de los estudiantes mejorar el contenido de sus trabajos y defenderlos oralmente en las jornadas. Esto significaba que los profesores tenían algo que enseñar a sus estudiantes (cómo escribir un trabajo, las características y la ejecución del género), pero los estudiantes tenían algo que mostrar (una hipótesis original en torno a la cual se construían sus investigaciones y trabajos). En consecuencia, el contrato didáctico tradicional -es decir, las expectativas que los profesores poseen e imparten de forma exclusiva los contenidos, mientras los estudiantes los reciben más o menos pasivamente- se rompió y modificó: hubo una renegociación de las expectativas entre el contenido que se debía enseñar (los profesores enseñaban a escribir una ponencia) y el ejercicio de las prácticas de escritura (los estudiantes ejercían la práctica de escritura de una manera relativamente autónoma, con el acompañamiento de un profesor-tutor).

En el ejemplo de Ramiro, puede observarse cómo el estudiante respondió preguntas críticas de sus compañeros y profesores. El Estudiante 2 hizo espontáneamente algunas preguntas que se le ocurrieron mientras escuchaba la presentación oral de Ramiro. Vale la pena mencionar que sólo unos pocos estudiantes habían leído de antemano las ponencias presentadas durante el evento científico. La mayoría de ellos sólo tuvo acceso a los 
resúmenes y escuchó las presentaciones orales. Ante esta pregunta de su compañero, Ramiro asumió la responsabilidad de fundamentar las afirmaciones hechas durante la presentación oral de su trabajo. Ramiro respondió al Estudiante 2 que la cita de los estudios de una autora coreana estaba relacionada con la necesidad de trabajar el espacio y las emociones desde una perspectiva literaria -y no psicológica-. El Estudiante 2 pareció satisfecho con la respuesta de Ramiro. Por lo menos, no hubo ninguna nueva pregunta, que indicase insatisfacción en este sentido. Sin embargo, en el turno [6], la profesora Silvana comenzó a discutir un quehacer del escritor (LERNER, 2001) relacionado con el campo académico: atenuar las afirmaciones, limitar su alcance y añadir algunos matices. Silvana consideró que Ramiro había construido su "nicho de investigación" de manera demasiado categórica y le advirtió del riesgo de hacerlo al escribir textos científicoacadémicos. Para ello, explicó que el espacio como valor simbólico había sido estudiado en el campo disciplinar de la Semiótica. En el turno 13, Ramiro cedió al cuestionamiento de la profesora Silvana, pero reafirmó su posición declarando: "Sí, principalmente quería subrayar, digamos, que el aspecto simbólico no sería central en mi obra, sino la emoción que proviene del aspecto simbólico (...) Pero es cierto que hay obras que no hemos tenido en cuenta”. Es decir, Ramiro aceptó la crítica e hizo una concesión, pero también reafirmó que el aporte original de su trabajo no consistía en mostrar el espacio como valor simbólico, sino el espacio y la emoción como símbolos.

En este extracto de la conversación, Ramiro defendió algo más que el nicho que había construido para su investigación, defendió su autonomía como lector y escritor académico. Ejerció su autoría: había elegido el contenido de su texto (los cuentos de Alejandro Nicolau) y, en consecuencia, podía construir una imagen de su público y de sí mismo como autor (ethos académico) que tenía una contribución original para hacer. Por lo tanto, en este extracto de la conversación podemos observar que el contrato didáctico tradicional, caracterizado por la asimetría en las relaciones entre profesores, estudiantes y conocimientos (BROUSSEAU, 2007; SENSEVY, 2011) dio lugar a un nuevo contrato en el que el profesor ciertamente sabía más sobre algo (el género ponencia), pero el estudiante, después de haber investigado y construido una hipótesis sobre un género que él mismo había elegido, sabía más sobre esa área particular del conocimiento (en el caso de Ramiro, la obra literaria de Alejandro Nicolau).

En cuanto al tiempo didáctico, se amplió ya que toda la discusión se basó en lo que se ha leído y escrito más allá del aula. Ramiro era consciente de que el género "ponencia" es un texto que se escribe con el objetivo de ser presentado oralmente ante un público más o menos especializado, que generalmente no lee el texto con antelación. Por lo tanto, toda su presentación consistió en hablar sobre lo escrito, en exponer oralmente sus hallazgos y su incipiente proceso de investigación.

Este caso de presentación y defensa de una ponencia no constituye ciertamente un ejemplo habitual de escritura para aprender en clase. Sin embargo, sí forma parte de una propuesta alternativa de enseñanza llevada deliberadamente a cabo por los profesores de Lingüística para utilizar la escritura como herramienta epistémica y como práctica situada en su asignatura. El trabajo con los estudiantes en sucesivas versiones de sus textos a lo largo de un cuatrimestre, dándoles la posibilidad de elegir el contenido y el corpus de análisis, orientándolos en el acercamiento gradual a un nuevo género discursivo, 
permitió que estos diálogos fueran auténticos: los autores y el público asumieron papeles activos en la progresión del conocimiento, se preguntaron y respondieron mutuamente como compañeros/colegas. En el siguiente subapartado, analizamos otra alternativa para trabajar con la escritura para aprender en las aulas universitarias y para ampliar el tiempo didáctico: el trabajo realizado en el caso Biología.

\section{Situación de discusión de textos frente a pares y docentes (Caso Biología)}

En Biología, la situación de "Discutir textos frente a pares y docentes" tuvo lugar al principio de todas las clases observadas. Este es un componente constitutivo e iterativo de este curso: una hora y media de cada clase se dedica a esta situación. Después de compartir y discutir en pequeños grupos las producciones escritas que trajeron los estudiantes, estos participan en una discusión plenaria con sus profesores y el resto de sus compañeros. Por lo tanto, escribir en casa tiene sentido porque sin ese trabajo previo los estudiantes no podrían entender o participar en estas discusiones con todo el grupo clase. Así, pues, primero al escribir y luego al discutir las ideas que han puesto sobre el papel, se espera que los estudiantes de esta clase asuman una posición y justifiquen las relaciones que establecen cuando se enfrentan a tareas que les demandan relacionar dos o más conceptos teóricos y vincularlos con situaciones de la vida cotidiana. En el siguiente párrafo, podemos ver una consigna con la que los estudiantes trabajaron en casa y en el aula. Esta les exigía analizar tres textos breves que trataban sobre ejemplos de las relaciones entre fenotipo, genotipo y ambiente.

\section{ACTIVIDAD 5}

Los siguientes textos describen situaciones en las que los conceptos de genotipo, fenotipo y ambiente están implícitos. Después de leerlos, haga las actividades.

[Los textos 1 y 2 fueron excluidos porque no se trabajan en el intercambio presentado].

\section{Texto 3}

La labor de los científicos ha puesto de manifiesto muchos aspectos del papel que desempeña la radiación solar en la incidencia anual de un número muy elevado de casos de cáncer de piel. Si la piel se expone a la luz del sol, acumulará alteraciones en la molécula de ADN (mutaciones) que se ven favorecidas por la radiación ultravioleta del espectro solar. Una célula puede caer en una multiplicación desenfrenada si una mutación transforma un gen normal en un promotor de crecimiento o inactiva un gen responsable de detener la división celular. También se sabe que un importante factor de riesgo es la asociación entre la piel blanca y la radiación solar intensa. Los australianos costeros de piel clara tienen la mayor incidencia de todos los cánceres de piel en el mundo, mientras que los aborígenes de piel más oscura casi nunca han tenido ese tipo de tumores.

Identifique en cada texto los tipos de interacciones presentes: genotipo-fenotipo; genotipo-ambiente; ambiente-genotipo o ambiente-fenotipo. 
1- Transcriba tres frases que refieran a algunas de las relaciones anteriores.

2- Indique, en cada caso, el tipo de relación;

4- Describa sus componentes (qué conceptos corresponderían al fenotipo, al genotipo o al ambiente).

3- ¿Por qué cree que no hemos mencionado ningún caso en el que se establezca la siguiente relación: un fenotipo que modifica al ambiente?

Esta consigna comienza con una pregunta abierta (lectura de tres textos con ciertas relaciones implícitas en ellos) y luego, en los puntos 1, 2, 3 y 4, profundiza en algunas especificaciones sobre la justificación de las relaciones encontradas. Es una tarea que presenta tres escenarios de la vida real interconectados, ya que todos ellos pueden referirse a los mismos conceptos teóricos. Después de completar las actividades en casa, los estudiantes comparten y discuten sus respuestas en pequeños grupos en la clase. Cada grupo designa un "portavoz" que habla en nombre de sus compañeros en la puesta en común con toda la clase. En este tipo de actividades de clase, no sólo los textos seleccionados por los profesores sino también las producciones escritas de los estudiantes se convierten en objeto de reflexión y debate conjunto. El siguiente extracto muestra cómo la profesora regresa al texto fuente, aquel que -en la consigna- buscaba que los estudiantes construyeran interpretaciones relevantes a través de la escritura, poniendo la "relectura cuidadosa" el centro de este intercambio.

[21] Profesor 1: Ahora, los que leyeron el texto 3 [se refiere a los dos grupos que designó previamente para trabajar con el texto 3], ¿qué relación encontraron? Es la más larga, ¿no?

[22] Profesor 2: Sí, es larga.

[23] Estudiante 3: Sí, aquí. Encontramos una respuesta: que la piel expuesta a la luz solar produce una mutación en el ADN, que es una relación ambiente-genotipo....

[34] Profesor 1: ¿Ambiente...?

[25] Estudiante 3: Genotipo. El ambiente sería la luz solar y el genotipo, la molécula de ADN.

[26] Profesor 1: ¿Qué hay de la molécula de ADN?

[27] Estudiante 3: Está alterada.

[28] Profesor 1: Si hay algo que no entienden sobre lo que estoy escribiendo en la pizarra, háganmelo saber. Sólo estoy tratando de abreviar, ¿Sí? Ehh... en la frase, vos decís que dice [dice] "si la piel se expone a la luz del sol, se acumularán alteraciones en la molécula de ADN (mutaciones)". ¿Es esa la frase?

[29] Estudiante 3: Sí.

[30] Profesor 1: ¿Están de acuerdo? ¿Qué hay del resto? ¿Qué opinan?

[31] Varios estudiantes: [inaudible].

[32] Profesor 1: ¿Está bien? Está hablando de un factor ambiental, los rayos UV en particular. No lo dice en esa frase, pero más tarde el texto lo menciona. Son los rayos UV favorecidos por la irradiación ultravioleta del espectro solar. ¿De acuerdo? Ese es un factor ambiental que está modificando directamente el material genético de las células de la piel, que son las más expuestas. ¿Verdad? ¿Es eso correcto? Bueno, esa relación está bien. Hay una mención explícita del genotipo. ¿Alguna otra [relación] encontrada? 
[33] Estudiante 4: Sí, ambiente-fenotipo.

[34] Profesor 1: [Escribiendo en la pizarra] Ambiente-fenotipo. ¿Y en qué frase o dónde, en qué parte del texto?

[35] Estudiante 4: Aquí dice que "la radiación solar produce cáncer de piel”.

[36] Profesor 1: ¿Así que sería el ambiente...?

[37] Estudiante 4: Fenotipo.

[38] Profesor 1: [Pausa] ¿Están de acuerdo?

[39] Varios estudiantes: Sí.

[40] Profesor 1: ¿Están todos de acuerdo en que el cáncer de piel está relacionado con el fenotipo?

[41] Estudiante 5: Entonces la modificación de la molécula de ADN es la receta y el pastel sería el cáncer de piel.

[42] Profesor 1: Exactamente, exactamente. ¿Sí? ¿Cuál es el problema en este caso particular? La luz ultravioleta está alterando mi receta.

[43] Estudiante 5: Claro, claro.

[44] Profesor 1: ¿Sí? ¿Está bien? Está haciendo cambios en los ingredientes, no sé, en los pasos a seguir, así que cuando quiero hacer el pastel, no lo hago bien.

En esta puesta en común con toda la clase, estudiantes y profesores hicieron uso de un quehacer del lector (LERNER, 2001) muy frecuente: releer el texto fuente para ajustar las interpretaciones y construcciones de significados. En este caso, volvieron al texto para buscar pistas sobre las posibles relaciones que se podrían dibujar entre el fenotipo, el genotipo y el ambiente basándose en el ejemplo del cáncer de piel. Este retorno al material escrito por parte del profesor para resolver las diferentes hipótesis construidas por los estudiantes está estrechamente ligado a la necesidad de sumergirse en un tema para tener más herramientas para escribir. La introducción de esta estrategia de lectura por parte del profesor impactó en la forma en que se manejaba el tiempo de enseñanza. Aunque la Profesora 1 comenzó por abordar el texto 3 de una manera bastante general (véase el turno 21), a medida que la conversación progresaba, con el texto en mano, guió a los estudiantes hacia interpretaciones más plausibles y sólidas (véanse los turnos 26 y 28, por ejemplo). Los estudiantes, inmersos en la práctica de la lectura de un texto difícil, adoptaron esta estrategia en su búsqueda de interpretaciones más acertadas. En el turno 41, el Estudiante 5 recurrió pertinentemente a una metáfora utilizada en una clase anterior, mientras que la Profesora 1, en los turnos 42 y 44, validó y profundizó el razonamiento planteado por su alumno: el genotipo era la información, la receta del pastel, por lo que el fenotipo y el ambiente eran las formas en que se llevaba a cabo esa receta particular.

La construcción del conocimiento fue de la mano de las preguntas de la profesora. En este sentido, los intercambios orales en esta aula terminaron siendo eminentemente radiales con la profesora en el centro (CAZDEN, 1998). Desde el turno 25 hasta el 28, la profesora interrogó a los estudiantes, buscando mayor precisión: “¿Qué pasa con la molécula de ADN?” o, más tarde, reforzó con preguntas corroborativas como “¿Están de acuerdo?" (turno 38). En una clase de primer año, masiva y heterogénea, la Profesora 1 insistió en involucrar a sus alumnos en lugar de limitarse a dar una clase magistral sobre los nuevos contenidos. La Profesora 1 logró esta tarea con relativo éxito: no sólo 
fue ella quien incluyó nuevos conocimientos en el intercambio, sino que los estudiantes también lo hicieron, básicamente, de dos maneras: (1) respondiendo a las preguntas de la Profesora 1 (turnos 23, 25, 27, 33, 35, 37 y 39); y (2) aportando conocimientos previos que les permitieran interpretar mejor los nuevos (turno 41). Así, cuando el Estudiante 5, en el turno 41, reflexionó "En otras palabras, la modificación de la molécula de ADN es la receta y el pastel sería el cáncer de piel”, pudo mostrar a su profesora no sólo que había entendido lo que había leído y lo que intentaba explicar, sino que también era capaz de relacionar esta comprensión -esos significados disciplinares construidos conjuntamentecon otros elaborados en clases anteriores. El Estudiante 5 ejerció, en el turno 41, otro quehacer lector frecuente: relacionar lo que se está leyendo con lo leído anteriormente, establecer relaciones entre los textos, "intertextualizar". Por lo tanto, cuando el Estudiante 5 volvió a una metáfora utilizada anteriormente para explicar (y explicarse) lo que intentaba comprender, puso en marcha otro aspecto de la práctica social de la lectura. La Profesora 1 comprendió este movimiento y lo retomó en sus siguientes intervenciones.

En resumen, en lo que respecta a las prácticas de lectura y escritura, este intercambio puso en marcha esencialmente dos quehaceres del lector (LERNER, 2001): 1) releer el texto de origen para ajustar las interpretaciones y 2) establecer relaciones entre este texto y otros previamente leídos. De este modo, en un intercambio dirigido por la profesora, sostenido por sus preguntas insistentes, corroborativas y repetitivas, los alumnos realizaron en clase una práctica de lectura que puso de relieve estas dos estrategias fundamentales de lectura. La profesora mostró la importancia de releer un texto al construir una interpretación e instó a los estudiantes a hacerlo en clase. Lo hizo precisamente pidiéndoles que señalaran los fragmentos específicos en los que encontraron las relaciones entre fenotipo, genotipo y ambiente (turnos 28, 32 y 34). Los estudiantes, en particular el Estudiante 5, subrayaron la necesidad de establecer relaciones entre esta y otras lecturas. El reto para los alumnos fue encontrar las relaciones pertinentes entre fenotipo, genotipo y ambiente, ofrecer nuevos ejemplos de las relaciones entre los conceptos en estudio. En este fragmento, vemos que defendieron dos tipos de relaciones: ambiente-genotipo (turnos 23 a 32) y ambiente-fenotipo (turnos 32 a 44). La Profesora 1 dirigió la clase, interrogó en momentos clave (turnos 26, 34 y 36) y alentó la participación cuando observó que las relaciones propuestas (ambientegenotipo y ambiente-fenotipo) habian sido comprendidas (turnos 32 y 44). A pesar de la forma radial que tomó el intercambio conversacional, fueron las intervenciones de los estudiantes las que hicieron posible que el profesor avanzara y validara las interpretaciones de aquellos que se acercaban al conocimiento disciplinar en cuestión.

\section{Conclusiones}

En este artículo se describen dos formas interrelacionadas pero diferentes de renegociar las responsabilidades hacia la construcción del conocimiento y de gestionar el tiempo didáctico cuando se escribe para aprender contenidos disciplinares.

En lo que respecta al tiempo didáctico, en estos casos, la incorporación de la escritura y la lectura fuera del aula sirvió como amplificador del espacio dedicado a la acción conjunta. Cuando se incorporó la escritura a las prácticas docentes como herramienta epistémica, se amplió el tiempo de enseñanza: un trabajo intenso dentro de la 
clase permitió a los alumnos trabajar de forma independiente en casa y, dialécticamente, utilizar ese trabajo realizado en sus hogares para participar en los debates al interior del aula. Esta modificación se produjo, en nuestros casos, dentro de dos situaciones: "Defender textos frente a pares y docentes (Lingüística)" y "Discutir textos frente a pares y docentes (Biología)".

En lo que respecta a la renegociación de las responsabilidades en la construcción del conocimiento, un fuerte énfasis en la organización de las clases en torno a la escritura permitió a los profesores y a los estudiantes trabajar colaborativamente e implementar estrategias conjuntas para avanzar con la progresión de los contenidos. En esta interacción de expectativas, los profesores de ambos casos preservaron la cláusula de reticencia (SENSEVY, 2011), para que sus estudiantes pudieran actuar motu proprio. Así, se renegoció el tradicional contrato didáctico: ni la progresión del conocimiento ni la responsabilidad de su producción recayó exclusivamente en los profesores, sino que se convirtió en una tarea cooperativa entre los participantes. Y esto sucedió dentro de la clase, entre otras cosas, porque los profesores hicieron posible -a partir del medio que configuraron- que los alumnos se pusieran a trabajar en casa, lejos de los confines del aula. El aula, entonces, se convirtió en un foro de debate, un espacio para el diálogo sobre lo que se había leído y escrito previamente (DYSTHE, 1996, 2012). Esta organización de la clase implicó abrazar una distribución más simétrica de los roles con respecto a la construcción del conocimiento disciplinar.

En resumen, nuestros casos destacan que "escribir para aprender" requiere que los profesores coloquen el trabajo con la lectura y la escritura en el centro de las actividades del aula. Al hacerlo, estas prácticas adquieren un significado genuino para sus estudiantes y les permiten participar críticamente en la construcción de conocimientos.

\section{Referencias}

ALVARAD0, Maite; CORTÉS, Marina. La escritura en la Universidad: repetir o transformar. Ciencias Sociales, Buenos Aires, v. 1., n. 43, p. 1-3, 2000.

ARNOUX, Elvira; ALVARAD0, Maite. La escritura en la lectura: apuntes y subrayado como huellas de representaciones de textos. In: MARTínEZ, María Cristina (coord.). Los procesos de la lectura y la escritura. Santiago de Cali: Universidad del Valle, 1997. p. 57-77.

ARNOUX, Elvira et al. El aprendizaje de la escritura en el ciclo superior. In: ARNOUX, Elvira (comp.). Adquisición de la escritura. Rosario: Juglaría, 1996. p. 57-63.

ARTIGUE, Michèle. Ingénierie didactique. Recherches en Didactique des Mathématiques, Grenoble, v. 9, n. 3, p. 283-307, 1990.

BARTON, David; HAMILTON, Mary. Understanding literacy as social practice. In: BARTON, David; HAMILTON, Mary (ed.). Local literacies: reading and writing in one community. London: Routledge, 1998. p. 3-22. 
BAZERMAN, Charles. Shaping written knowledge: the genre and activity of the experimental article in science. Madison: University of Wisconsin Press, 1988.

BAZERMAN, Charles; PRIOR, Paul. What writing does and how it does it: an introduction to analyzing texts and textual practices. New Jersey: Lawrence Erlbaum, 2004.

BAZERMAN, Charles et al. Reference guide to writing across the curriculum. West Lafayette: Parlor Press, 2005.

BROUSSEAU, Guy. Iniciación al estudio de la teoría de las situaciones didácticas. Buenos Aires: Libros del Zorzal, 2007.

CAMILLONI, Alicia. Justificación de la didáctica. In: CAMILLONI, Alicia et al., El saber didáctico. Buenos Aires: Paidós, 2012. p. 19-22.

CARLINO, Paula. Alfabetización académica diez años después. Revista Mexicana de Investigación Educativa, México, DC, v. 18, n. 57, p. 355-381, 2013.

CARLINO, Paula. Escribir, leer y aprender en la universidad: una introducción a la alfabetización académica. Buenos Aires: Fondo de Cultura Económica de Argentina, 2005.

CARLINO, Paula. Estudiar, escribir y aprender en universidades australianas. Textura, Maturín, v. 6, n. 9, p. 11-33, 2007.

CARLINO, Paula. Leer, escribir y aprender en la universidad: cómo lo hacen en Australia y por qué. Investigaciones en Psicología, Buenos Aires, v. 7, n. 2, p. 43-61, 2002.

CARLINO, Paula et al. Leer y escribir para aprender en las diversas carreras y asignaturas de los IFD que forman a profesores de enseñanza media: concepciones y prácticas declaradas de los formadores de docentes. Buenos Aires: Ministerio de Educación de la Nación, 2013.

CARTER, Michael; FERZLI, Miriam; WIEBE, Eric N. Writing to learn by learning to write in the disciplines. Journal of Business and Technical Communication, lowa, v. 21, n. 3, p. 278-302, 2007.

CARTOLARI, Manuela; MOLINA, María Elena. Modos de leer y escribir en historia: percepciones de alumnos y profesores de la formación docente inicial. Redu - Revista de Docencia Universitaria, Valencia, v. 13, n. 3, p. 235-263, 2016.

CAZDEN, Courtney. El discurso en el aula: el lenguaje de la enseñanza y del aprendizaje. Barcelona: Paidós, 1991.

COLOMBO, Laura. The role of social relations in the making of a doctoral thesis. International Journal of Educational Research and Innovation, Sevilla, v. 2, n. 10, p. 366-378, 2018.

CONDON, William; RUTZ, Carol. A Taxonomy of writing across the curriculum programs: evolving to serve broader agendas. College Composition and Communication, Illinois, v. 64, n. 2, p. 357-382, 2012. 
CRESWELL, John W. Qualitative inquiry \& research design: choosing among five approaches. 2. ed. Thousand Oaks: Sage, 2007.

DAVINI, María Cristina. Métodos de enseñanza. Buenos Aires: Santillana, 2008.

DI STEFANO, Mariana; PEREIRA, Cecilia. La enseñanza de la lectura y escritura en el nivel superior: procesos, prácticas y representaciones sociales. In: CARLINO, Paula (coord.). Leer y escribir en la universidad. Buenos Aires: Lectura y Vida; International Reading Association, 2004. p. 23-39. (Textos en contexto; n. 6).

DYSTHE, Olga. The multivoiced classroom: interactions of writing and classroom discourse. Written Communication, Thousand Oaks, v. 13, n. 3, p. 385-425, 1996.

DYSTHE, Olga. Multivoiced classrooms in higher education academic writing. In: CASTELLÓ, Montserrat; DONAHUE, Christiane (ed.). University writing: selves and texts in academic societies. London: Emerald, 2012. p. 201-216.

FERNÁNDEZ, Graciela; IZUZQUIZA, Viviana; LAXALT, Irene. El docente universitario frente al desafío de enseñar a leer. In: CARLINO, Paula (coord.). Leer y escribir en la universidad. Buenos Aires: Lectura y Vida; International Reading Association, 2004. p. 95-110. (Textos en contexto; n. 6).

IGLESIA, Patricia; DE MICHELI, Ana. Leer textos de biología en el primer año de la universidad: ¿es un saber construido 0 una práctica a enseñar? Enseñanza de las Ciencias, Barcelona, v. 1, n. 8, p. 817-820, 2009. Congreso Internacional sobre Investigación en Didáctica de las Ciencias.

LANGER, Judith; APPLEBEE, Arthur. How writing shapes thinking: a study of teaching and learning. Urbana: National Council of Teachers of English, 1987.

LEA, Mary; STREET, Brian. Student writing in higher education: an academic literacies approach. Studies in Higher Education, London, v. 23, n. 2, p. 157-172, 1998.

MAXWELL, Joseph. Qualitative research design: an interactive approach. London: Sage, 2005.

MAXWELL, Joseph; MILLER, Barbara. Categorizing and connecting strategies in qualitative data analysis. In: LEAVY, Patricia; HESSE-BIBER, Sharlene (ed.). Handbook of emergent methods. New York: Guilford Press, 2008. p. 85-106.

MOLINA, María Elena; CARLINO, Paula. Writing as a way of participating in knowledge construction in two Argentine University Classrooms. In: BAZERMAN, Charles et al. (ed.). Knowing writing: writing research across borders. [Conocer la escritura: investigación más allá de la frontera]. Bogotá: Pontificia Universidad Javeriana, 2019. p. 237-256.

NATALE, Lucía. La construcción del resumen: una propuesta didáctica basada en la lingüística sistémico funcional. In: CONGRESO INTERNACIONAL "DEBATES ACTUALES: LAS TEORÍAS CRÍTICAS DE LA LITERATURA Y LA LINGÜÍSTICA", 2004, Buenos Aires. Anais... Buenos Aires: Universidad de Buenos Aires, 2004. p. 39-46.

PADILLA, Constanza. Escritura y argumentación académica: trayectorias estudiantiles, factores docentes y 
contextuales. Magis, Bogotá, v. 5, n. 10, p. 31-57, 2012.

PADILLA, Constanza; ÁVILA, Ana María; LOPEZ, Esther. ¿Cómo preparamos a los estudiantes universitarios para abordar textos académicos? Lectura y escritura: caminos para la construcción del mundo. Catamarca: Unesco, 2007. CD-Rom. Editorial científica universitaria de la UNC. Cátedra UNESCO.

PATTON, Michael. Qualitative evaluation and research methods. Beverly Hills: Sage, 1991. p. 169-186.

PIACENTE, Telma; TITTARELLI, Ana María. ¿Alfabetización universitaria? In: JORNADAS DE INVESTIGACIÓN EN PSICOLOGÍA, 10., 2003, Buenos Aires. Memorias de... t. I. Buenos Aires: Universidad de Buenos Aires, 2003. p. 290-292.

RICKENMANN, René. Metodologías clínicas de investigación en didácticas y formación del profesorado: un estudio de los dispositivos de formación en alternancia. In: CONGRESO INTERNACIONAL DE INVESTIGACIÓN, EDUCACIÓN Y FORMACIÓN DOCENTE, 2006, Medellín. Actas del... Medellín: Universidad de Antioquia, 2006. p. 1-16.

ROSLI, Natalia; CARLINO, Paula; CARTOLARI, Manuela. Intervenciones docentes para leer en ciencias sociales: exploración en un quinto año de secundaria con alumnos de sectores socioeconómicos desfavorecidos. Legenda, Mérida, v. 17, n. 16, p. 1-23, 2013.

RUSSELL, David. Contradictions regarding teaching and writing (or writing to learn) in the disciplines: what we have learned in the USA. Redu - Revista de Docencia Universitaria, Valencia, v. 11, n.1, p. 161-181, 2013.

SENSEVY, Guy. Des catégories pour décrire et comprendre l'action didactique. In: SENSEVY, Guy; MERCIER, Alan (ed.). Agir ensemble: l'action didactique conjointe du professeur et des élèves. Rennes: Presses Universitaires de Rennes, 2007. p. 5-34.

SENSEVY, Guy. Le sens du savoir: éléments pour une théorie de l'action conjointe en didactique. Bruxelles: Boeck, 2011.

STAKE, Robert. Investigación con estudio de casos. Madrid: Morata, 1998.

THAISS, Christopher; PORTER, Tara. The State of WAC/WID in 2010: methods and results of the U.S. survey of the International WAC/WID mapping project. College Composition and Communication, Illinois, v. 61, n. 3, p. 534-570, 2010.

VÁZQUEZ, Alicia; MIRAS, Mariana. Cómo se representan estudiantes universitarios las tareas de escritura. REUNIÓN INTERNACIONAL "MENTE Y CULTURA: CAMBIOS REPRESENTACIONALES EN EL APRENDIZAJE", 2004, Madrid. Actas electrónicas de la... Madrid: Universidad Nacional del Comahue; Universidad Autónoma de Madrid, 2004. p. 1-17.

VÁZQUEZ, Alicia et al. Enseñar a escribir en la universidad: análisis de los cambios en las estrategias de producción de textos. In: JORNADAS DE INVESTIGACIÓN EN PSICOLOGÍA, 10., 2003, Buenos Aires. Memorias de las... t. I. Buenos Aires: Universidad de Buenos Aires, 2003. p. 322-325. 
Recibido en: 17.04.2020

Revisado en: 03. 06. 2020

Aprobado en: 30. 06. 2020

María Elena Molina es Doctora en Humanidades (Universidad Nacional de Tucumán). Jefe de Trabajos Prácticos de Didáctica General en la Universidad Nacional del Sur (Bahía Blanca, Argentina). Becaria postdoctoral de CONICET (Consejo Nacional de Investigaciones Científicas y Técnicas, Argentina). Miembro de GICEOLEM (Grupo para la Inclusión y la Calidad Educativas a través de Ocuparnos de la Lectura y la Escritura en todas las Materias), equipo de investigación dirigido por la Dra. Paula Carlino (CONICET).

Laura Marina Colombo es Doctora en Lenguaje, Literacidad y Cultura (University of Maryland, Baltimore County). Investigadora Adjunta de CONICET (Consejo Nacional de Investigaciones Científicas y Técnicas, Argentina). Miembro de GICEOLEM (Grupo para la Inclusión y la Calidad Educativas a través de 0cuparnos de la Lectura y la Escritura en todas las Materias), equipo de investigación dirigido por la Dra. Paula Carlin (CONICET). 